\title{
Prioritization of Influence Factors for Selecting E-Learning Systems
}

\author{
Ali Karasan ${ }^{1(\bowtie)}(1)$ and Melike Erdogan ${ }^{2}(\mathbb{C}$ \\ 1 Department of Industrial Engineering, Yildiz Technical University, \\ Besiktas, 34347 Istanbul, Turkey \\ akarasan@yildiz.edu.tr \\ 2 Department of Industrial Engineering, Duzce University, \\ Konuralp, 81620 Duzce, Turkey \\ melikeerdogan@duzce.edu.tr
}

\begin{abstract}
COVID-19 pandemic affects not only daily life activities but also traditional education systems. Based on the current developments, to stick by their academic calendars, most of the educational institutions continue their classes via online channels. Since the selection of the most appropriate e-learning platform depends on multi-criteria, the evaluation of this selection process can be dealt with decision support systems. In this study, cognitive mapping extended with intuitionistic fuzzy sets is introduced for prioritizing the e-learning platform selection factors under fuzzy environment based on the multi-expert judgments. Based on the results, infrastructure and ease of use are determined as the most effective factors. For further studies, a sensitivity analysis based on the initial vector determination can be studied to check its effect on the outputs.
\end{abstract}

Keywords: E-learning - Intuitionistic fuzzy sets - Cognitive maps · Decision making

\section{Introduction}

One of the most important sectors affected by the extraordinary situations experienced by countries is the education. In cases of war, epidemic disease, earthquake, etc., students become unable to receive education and if the effects of these situations continue for a long time, both students and all elements of the education system will be irreversibly damaged. Due to the COVID-19 pandemic that has been happening all over the world recently, countries have stopped taking face-to-face training activities at all levels. The absence of a clear prediction on the ending date of the pandemic also required action to be taken as soon as possible on behalf of educational activities. One of the precautions that can be taken in order to prevent educational activities from disrupting is to apply to e-learning platforms.

Today, most of the educational institutions continue their classes via online channels to stick by their academic calendars. E-learning is the using of internet

(C) The Editor(s) (if applicable) and The Author(s), under exclusive license

to Springer Nature Switzerland AG 2021

C. Kahraman et al. (Eds.): INFUS 2020, AISC 1197, pp. 550-556, 2021.

https://doi.org/10.1007/978-3-030-51156-2_63 
so as to learn specific information and content of students [1]. As the synonym of e-learning, web-based learning, online learning, course-learning, virtual learning, and digital collaboration learning are used [1]. E-learning is an effective learning process created by combining digitally presented content with learning support services [2]. E-learning is a very useful tool to ensure the continuity of teaching and learning in order to allow students to have distance education. It not only provides continuity of education, but also promotes to develop at the institutional and personal level, including both faculty and students [3]. Universities have also started to use e-learning platforms due to the extraordinary situation such as COVID19 pandemic in the world nowadays, but each university can have different expectations in terms of the technical and flexibility features of these platforms. For this reason, choosing the e-learning platform that meets the expectations best in such an environment is a process that many different criteria should be considered.

The concept of e-learning is a concept that attracts a lot of attention not only in practice but also in the literature. The selection of the most suitable e-learning platform is a subject previously addressed by multi-criteria decision making methods. One of the outstanding papers in this regard, paper of Alptekin and Karsak which is proposed a hybrid model in the selection of e-learning applications by the universities in Turkey [2]. In the proposed approach, the QFD method was used to allocate resources and coordinate functions according to user expectations, and fuzzy regression was used to determine the functional relationship between customer expectations and product features. Begicevic et al. evaluated e-learning practices in 4 stages as intelligence, design, choice and implementation and applied AHP method to determine the best alternative in the selection stage [3]. Bhuasiri et al. addressed the determination of critical success factors affecting the acceptance of e-learning systems [4]. Relative importance of these factors was calculated by AHP with responses from 76 users for information and communications technology experts' perspective and faculty members perspective separately. Chao and Chen identified five main criteria and sixteen sub-criteria for a successful electronic learning application and used consistent fuzzy preference relations in AHP to determine the relative importance of these criteria relative to each other [5]. Bo and Peng identified the main factors to measure the effectiveness of network education in China and calculated index weights using fuzzy AHP [6]. In addition to these papers, the study of Zare et al. could be accepted as the main reference for researchers who would study on decision making for e-learning with their detailed literature review [1]. However, unlike all these studies, a different perspective is provided for the first time by using the intuitionistic fuzzy cognitive mapping method. Intuitionistic fuzzy cognitive mapping is applied and it is determined which criteria should be taken into consideration primarily for the universities that want to select an e-learning platform for the distance education during the pandemic period.

The rest of the paper is organized as follows. Section 2 presents the applied methodology. In Sect.3, the application is carried out. The paper ends with obtained results and future work suggestions. 


\section{Methodology}

Since the prioritization problem consists of multi evaluation criteria and many alternatives together with human knowledge on the operation of the system, the structure of the problem can be constructed by using expert judgments. The cognitive maps were firstly introduced by Axelrod to represent interrelationships and their corresponded binary values [7] based on the human evaluations. After that, Kosko constructed fuzzy cognitive maps to increase its ability by representing not only cardinal information but also vague judgments [8]. In the constructed environment, construction methodologies rely on the exploitation of experts' experience on system's model and behavior [9]. Therefore, constructing a scale based on the linguistic terms is an efficient way of representing the human knowledge. On the other hand, experts can be hesitant while express their judgments. In these systems where there is no exact determinacy about the evaluations, the most appropriate way to use the data is to formulate hesitancy and integrate it to mathematical formulations. In our paper, Intuitionistic fuzzy sets are applied to extend cognitive maps to use this advantage while deal with the hesitant information [9].

Step 1. Assign the linguistic terms for membership functions based on the scale given in Table 1.

Table 1. Linguistic terms and their corresponded membership functions

\begin{tabular}{l|l}
\hline Linguistic term & Corresponded triangular membership function \\
\hline Neg Very Strong -VS & $(-1,-1,-0.7)$ \\
\hline Neg Strong $-\mathrm{S}$ & $(-1,-0.7,-0.5)$ \\
\hline Neg Medium - M & $(-0.7,-0.5,-0.3)$ \\
\hline Neg Weak - V & $(-0.5,-0.3,-0.1)$ \\
\hline Neg Very Weak -VW & $(-0.2,-0.1,0)$ \\
\hline No Relation N & $(-0.1,0,0.1)$ \\
\hline Pos Very Strong VW & $(0,0.1,0.2)$ \\
\hline Pos Strong V & $(0.1,0.3,0.5)$ \\
\hline Pos Medium M & $(0.3,0.5,0.7)$ \\
\hline Pos Weak S & $(0.5,0.7,1)$ \\
\hline Pos Very Weak VS & $(0.7,1,1)$ \\
\hline
\end{tabular}

Step 2. Determine the hesitancy of the membership functions based on the scales given in Table 2 .

Step 3. Convert linguistic terms to obtain triangular membership and hesitancy functions.

Step 4. Construct the weight matrix. 
Table 2. Linguistic terms and their corresponded hesitancy functions

\begin{tabular}{l|l}
\hline Linguistic term & Corresponded hesitancy function \\
\hline Very Low VL & $(0,0.1,0.2)$ \\
\hline Low L & $(0.1,0.2,0.35)$ \\
\hline Medium M & $(0.25,0.4,0.6)$ \\
\hline High H & $(0.5,0.65,0.8)$ \\
\hline Very High VH & $(0.7,0.9,1)$ \\
\hline
\end{tabular}

Step 5. Compute the weights by using Eq. (1).

$$
s_{i}^{k+1}=f\left(s_{i}^{k}+\sum_{\substack{j=1 \\ j \neq i}}^{N} s_{j}^{k} \cdot w_{j i}^{\mu}-s_{j}^{k} \cdot w_{j i}^{\pi}\right)
$$

where $f(x)=\frac{1}{1+e^{-x}}$. The iteration is run until all the weights are converged or a certain number of iterations is reached.

Step 6. Obtain the aggregated weights with respect to decision makers.

\section{Application}

Through the determination of the most influenced criteria for the e-learning systems, 14 criteria are determined. 3 decision makers evaluated the system and constructed to interrelationships. List of the criteria is given in Table 3 .

Table 3. List of the criteria

\begin{tabular}{l|l}
\hline List of the criteria & \\
\hline C1-Ease of use [10] & C8-Pricing [11] \\
\hline C2-Ease of exchanging learning with the others [10] & C9-Flexibility [11] \\
\hline C3-Capability of controlling learning progress [10] & C10-Reporting [12] \\
\hline C4-Network infrastructure [3] & C11- Access (time \& place) [11] \\
\hline C5-Availability of technical support staff [3] & C12-Security \& privacy [11] \\
\hline C6-Exam management system [3] & C13-Trialability [11] \\
\hline C7-Video \& audio streaming [3] & C14-Interactivity level [11] \\
\hline
\end{tabular}


Evaluations of the decision maker 1 is given in Table 4 .

Table 4. Decision maker 1 evaluations

\begin{tabular}{|c|c|c|c|c|c|c|c|c|c|c|c|c|c|c|c|c|c|c|}
\hline & $\mathrm{P}$ & & C1 & $\mathrm{C} 2$ & C3 & $\mathrm{C} 4$ & c & 5 & C6 & & C8 & & $\mathrm{C} 1 \mathrm{C}$ & & C11 & C12 C13 & & C14 \\
\hline $\mathrm{P}$ & $\mathrm{N}$ & $\mathrm{VL}$ & $\mathrm{N}$ & VLN & VLNVL & $\mathrm{N}$ & VLN & $\mathrm{v}$ VI & $\mathrm{LN}$ & VL & $\mathrm{N} V L \mathrm{~N}$ & VL & $\mathrm{N} V L \mathrm{~N}$ & $\mathrm{VL}$ & $\mathrm{N}$ & VLN|VLN & $\mathrm{VL}$ & $\mathrm{N} \mid \mathrm{VL}$ \\
\hline $\mathrm{C} 1$ & $-\mathrm{S}$ & $\mathrm{L}$ & $\mathrm{N}$ & VLN & VLNVL & $\mathrm{N}$ & VLN & $\mathrm{N}$ VI & $\mathrm{LN}$ & VL & $\mathrm{N} V \mathrm{VL}-\mathrm{VW}$ & $\mathrm{L}$ & $\mathrm{N} V \mathrm{VL}-\mathrm{S}$ & $\mathrm{VH}$ & $\mathrm{N}$ & VLNVLS & $\mathrm{H}$ & $\mathrm{NVL}$ \\
\hline $\mathrm{C} 2$ & $\mathrm{~N}$ & $\mathrm{VL}$ & $\mathrm{N}$ & VLN & VLNVL & $\mathrm{N}$ & VLN & $\mathrm{N}$ VI & $\mathrm{L} \mathrm{N}$ & VL & NVLN & $\mathrm{VL}$ & NVLN & $\mathrm{VL}$ & $\mathrm{N}$ & VLNVL-S & $\mathrm{H}$ & NVL \\
\hline C3 & $\mathrm{N}$ & $\mathrm{VL}$ & $\mathrm{N}$ & VLN & VLNVL & & VLN & $\mathrm{N}$ VI & $\mathrm{L} N$ & VL & NVLN & $\mathrm{VL}$ & NVLN & $\mathrm{VL}$ & $\mathrm{N}$ & VLNVL-M & $\mathrm{VH}$ & $\mathrm{N} V \mathrm{VL}$ \\
\hline $\mathrm{C} 4$ & M & L & $\mathrm{N}$ & VLN & VLNVL & $\mathrm{N}$ & VLN & $\mathrm{N}$ VI & $\mathrm{L} N$ & VL & NVLN & $\mathrm{VL}$ & $\mathrm{NVLN}$ & $\mathrm{VL}$ & $\mathrm{N}$ & VLNVLN & $\mathrm{VL}$ & NVL \\
\hline C5 & $\mathrm{V}$ & $\mathrm{H}$ & $\mathrm{N}$ & VLN & VLNL & VW & $\mathrm{VLN}$ & $\mathrm{N}$ VI & $\mathrm{L} N$ & VL & NVLN & $\mathrm{VL}$ & $\mathrm{NVLN}$ & $\mathrm{VL}$ & $\mathrm{N}$ & VLNVLN & $\mathrm{VL}$ & $\mathrm{NVL}$ \\
\hline C6 & $-\mathrm{VS}$ & M & $\mathrm{N}$ & $\mathrm{VLN}$ & $\mathrm{VLNVH}$ & $\mathrm{V}$ & VLN & $\mathrm{N}$ VI & $\mathrm{L} N$ & VL & & $\mathrm{VL}$ & $\mathrm{NVLN}$ & $\mathrm{VL}$ & $\mathrm{N}$ & VLNVLN & $\mathrm{VL}$ & NVL \\
\hline $\mathrm{C} 7$ & $-\mathrm{VW}$ & $\mathrm{VH}$ & $\mathrm{N}$ & VLN & VLNVL & VS & VLN & $\mathrm{N}$ VI & $\mathrm{L}-\mathrm{VS}$ & $\mathrm{VH}$ & NVLN & $\mathrm{VL}$ & $\mathrm{N} V \mathrm{~L}-\mathrm{V}$ & $\mathrm{VH}$ & $\mathrm{N}$ & VLNVLS & $\mathrm{VL}$ & NVL \\
\hline $\mathrm{C} 8$ & M & $\mathrm{L}$ & $\mathrm{N}$ & VLN & VLNVL & $\mathrm{N}$ & VLN & $\mathrm{N}$ VI & $\mathrm{L} \mathrm{N}$ & VL & NVLN & $\mathrm{VL}$ & NVLV & $\mathrm{VL}$ & N & VLNVL-S & $\mathrm{VL}$ & $\mathrm{NVL}$ \\
\hline C9 & $-\mathrm{S}$ & M & $\mathrm{N}$ & VLN & VLNVL & $\mathrm{N}$ & VLN & $\mathrm{N}$ VI & $\mathrm{L} \mathrm{N}$ & VL & NVLN & $\mathrm{VL}$ & $\mathrm{NVL}-\mathrm{M}$ & $\mathrm{VH}$ & $\mathrm{N}$ & VLNVL-VW & $\mathrm{VH}$ & $\mathrm{NVL}$ \\
\hline $\mathrm{C} 10$ & & $\mathrm{M}$ & $\mathrm{N}$ & VLN & VLNVL & & VLN & $\mathrm{N}$ VI & $\mathrm{L} \mathrm{N}$ & VL & $\mathrm{N} V \mathrm{LL}$ & $\mathrm{VL}$ & NVLN & $\mathrm{VL}$ & $\mathrm{N}$ & VLNVLN & $\mathrm{VL}$ & $\mathrm{NVL}$ \\
\hline $\mathrm{C} 11$ & & $\mathrm{VH}$ & $\mathrm{N}$ & VLN & VLNVL & & VLN & $\mathrm{N} \mathrm{VI}$ & & VL & $\mathrm{NVLN}$ & $\mathrm{VL}$ & $\mathrm{NVLN}$ & $\mathrm{VL}$ & $\mathrm{N}$ & VLNVLN & $\mathrm{VL}$ & $\mathrm{NVL}$ \\
\hline $\mathrm{C} 12$ & & $\mathrm{VH}$ & $-\mathrm{V}$ & $\mathrm{M} N$ & VLNVL & N & $\mathrm{VL}-$ & & $\mathrm{HN}$ & VL & NVLN & $\mathrm{VL}$ & $\mathrm{NVLN}$ & $\mathrm{VL}$ & VW & $\mathrm{VLN}$ VLN & $\mathrm{VL}$ & NVL \\
\hline $\mathrm{C} 13$ & & $\mathrm{~L}$ & $-\mathrm{VW}$ & $\mathrm{VL}-\mathrm{VW}$ & VLNVL & $\mathrm{N}$ & VLS & L & $\mathrm{N}$ & VL & NVLN & $\mathrm{VL}$ & NVLN & $\mathrm{VL}$ & $\mathrm{N}$ & VLNVLN & $\mathrm{VL}$ & $\sqrt{V L}$ \\
\hline $\mathrm{C} 14$ & & $\mathrm{~L}$ & $-\mathrm{VW}$ & \begin{tabular}{l|l}
$\mathrm{H}$ & $\mathrm{N}$ \\
\end{tabular} & VLNVL & & VLN & $\mathrm{N} V \mathrm{VI}$ & & VL & NVLN & $\mathrm{VL}$ & $\mathrm{N} V L \mathrm{~N}$ & $\mathrm{VL}$ & $\mathrm{N}$ & VLN/VLN & $\mathrm{VL}$ & $\mathrm{NVL}$ \\
\hline
\end{tabular}

Evaluations of the decision maker 2 is given in Table 5 .

Table 5. Decision maker 2 evaluations

\begin{tabular}{|c|c|c|c|c|c|c|c|c|c|c|c|c|c|c|c|c|c|c|c|c|c|c|c|c|c|c|}
\hline & P & & C1 & & $\mathrm{C} 2$ & C3 & C4 & & C5 & & C6 & & $\mathrm{C} 7$ & & C8 & & C10 & & C11 & & C12 & & C13 & & C14 & \\
\hline $\mathrm{P}$ & $\mathrm{N}$ & VL & $\mathrm{N}$ & $\mathrm{VL}$ & $\mathrm{N}$ & VLN & VL $N$ & $\mathrm{VL}$ & & $\mathrm{VL}$ & & VL $\mathrm{N}$ & & $\mathrm{VL} / \mathrm{I}$ & $\mathrm{N}$ & $\mathrm{VL}$ & NVL $N$ & & & $\mathrm{VL}$ & $\mathrm{N}$ & VL I & $\mathrm{N}$ & ${ }_{\mathrm{L}}^{\mathrm{L}} \mathbf{\mathrm { N }}$ & $\mathrm{VI}$ & $V \mathrm{~L}$ \\
\hline C1 & $-\mathrm{S}$ & M & $\mathrm{N}$ & $\mathrm{VL}$ & $\mathrm{N}$ & $\mathrm{VL} N$ & VL $N$ & $\mathrm{VL}$ & $\mathrm{N}$ & $\mathrm{VL}$ & & $\left.\mathrm{VL}\right|_{\mathrm{N}}$ & & $\mathrm{VL}$ & $-\mathrm{VW}$ & L & $\mathrm{N} / \mathrm{VL}-\mathrm{S}$ & & & VL & $\mathrm{N}$ & VL & & ${ } \mathrm{H} \mid \mathrm{N}$ & & $V \mathrm{~L}$ \\
\hline $\mathrm{C} 2$ & $\mathrm{~N}$ & VL & $\mathrm{N}$ & $\mathrm{VL}$ & $\mathrm{N}$ & VL $N$ & VL $N$ & $\mathrm{VL} \mid$ & & $\mathrm{VL}$ & & $\mathrm{VL} \wedge$ & & $\mathrm{VL} / \mathrm{I}$ & $\mathrm{N}$ & $\mathrm{VL} \mid$ & $\mathrm{N}|\mathrm{VL}| \mathrm{N}$ & VL & & VL & $\mathrm{N}$ & $\mathrm{VL}$ & \begin{tabular}{l|l}
$-\mathrm{S}$ & $\mathrm{L}$
\end{tabular} & $\mathrm{N}$ & V & $V L$ \\
\hline C3 & $\mathrm{N}$ & VL & $\mathrm{N}$ & VL & $\mathrm{N}$ & $\mathrm{VL} N$ & $\mathrm{VL} N$ & $\mathrm{VL}$ & & $\mathrm{VL}$ & & $\left.\mathrm{VL}\right|_{\mathrm{N}}$ & & $\mathrm{VL}$ & $\mathrm{N}$ & VL & $\mathrm{N} V \mathrm{VL}$ & VL & & $\mathrm{VL}$ & $\mathrm{N}$ & $\mathrm{VL}$ & $-\mathrm{M} L$ & $\mathrm{~N}$ & $\mathrm{VI}$ & $V \mathrm{~L}$ \\
\hline $\mathrm{C} 4$ & $\mathrm{~N}$ & M & $\mathrm{N}$ & $\mathrm{VL}$ & $\mathrm{N}$ & VL $N$ & VL $N$ & $\mathrm{VL}$ & & $\mathrm{VL}$ & & $\mathrm{VL}$ & $-\mathrm{M}$ & $\mathrm{VL}$ & $\mathrm{N}$ & VL & $\mathrm{N} V \mathrm{VL}$ & VL & & $\mathrm{VL}$ & $N$ & $\mathrm{VL}$ & $\mathrm{N}$ & $V L N$ & & $V L$ \\
\hline C5 & $\mathrm{V}$ & $\mathrm{VL}$ & $\mathrm{N}$ & VL & $\mathrm{N}$ & $\mathrm{VL} N$ & VW & $\mathrm{H}$ & $\mathrm{N}$ & VL & & $\mathrm{VL} / \mathrm{N}$ & & $\mathrm{VL}$ & $N$ & $\mathrm{VL}$ & $\mathrm{N} V L \mathrm{~N}$ & VL & $\mathrm{N}$ & $\mathrm{VL}$ & $-\mathrm{VS}$ & $\mathrm{VH}$ & $N$ & \begin{tabular}{l|l}
$V L$ & $\mathrm{~N}$
\end{tabular} & V & $V L$ \\
\hline C6 & $-\mathrm{VW}$ & $\mathrm{VH}$ & $\mathrm{N}$ & $\mathrm{VL}$ & $\mathrm{N}$ & $\mathrm{VL} N$ & $\mathrm{VHV}$ & $\mathrm{VL}$ & N & VL & & $\mathrm{VL} / \mathrm{N}$ & & $\mathrm{VL}$ & $\mathrm{N}$ & VL & $\mathrm{N} V \mathrm{~L} N$ & VL & $-\mathrm{VS}$ & \begin{tabular}{|l|l}
$\mathrm{S}$ \\
\end{tabular} & $\mathrm{N}$ & & $\mathrm{N}$ & $\mathrm{VL} N$ & & $V \mathrm{~L}$ \\
\hline C7 & $\mathrm{N}$ & L & $\mathrm{N}$ & $\mathrm{VL}$ & & $\mathrm{VL} N$ & VL VS & $\mathrm{H}$ & N & VL & $-\mathrm{VS}$ & $\mathrm{M}$ & $\mathrm{N}$ & $\mathrm{VL} / \mathrm{I}$ & $\mathrm{N}$ & VL & $\mathrm{N} V \mathrm{~L}-\mathrm{V}$ & & & $\mathrm{VL}$ & $\mathrm{N}$ & & M L & . & $-\mathrm{MH}$ & \\
\hline $\mathrm{C} 8$ & $\mathrm{~N}$ & $\mathrm{VL}$ & $\mathrm{N}$ & $\mathrm{VL}$ & & $\mathrm{VL} M$ & $\mathrm{I} \mathrm{VL} \mathrm{N}$ & $\mathrm{VL}$ & $\mathrm{N}$ & VL & & $\mathrm{VL} / \mathrm{S}$ & & $\mathrm{VL}$ & $\mathrm{N}$ & $\mathrm{VL}$ & $\mathrm{NVL}-\mathrm{VW}$ & $\mathrm{VL}$ & $\mathrm{N}$ & $\mathrm{VL}$ & $\mathrm{N}$ & $\mathrm{VL}$ & $\mathrm{N}$ & \begin{tabular}{l|l}
$V L$ & $N$ \\
\end{tabular} & & $V \mathrm{~L}$ \\
\hline C9 & $-S$ & $\mathrm{VL}$ & $\mathrm{N}$ & $\mathrm{VL}$ & & $\mathrm{VLN}$ & VL $N$ & $\mathrm{VL}$ & & VL & & $\mathrm{VL} /$ & & $\mathrm{VL}$ & $\mathrm{N}$ & VL & NVL VW & L & $\mathrm{N}$ & $\mathrm{VL}$ & $\mathrm{N}$ & & $\mathrm{N}$ & $V L N$ & & $V \mathrm{~L}$ \\
\hline $\mathrm{C} 1 \mathrm{C}$ & & M & V & $\mathrm{VL}$ & & $\mathrm{VL} N$ & VL $N$ & $\mathrm{VL}$ & $\mathrm{N}$ & VL & & $\mathrm{VL} / \mathrm{N}$ & & $\mathrm{VL}$ & $-\mathrm{M}$ & $\mathrm{VL}$ & $\mathrm{N} V \mathrm{~L} N$ & VL & & $\mathrm{VL}$ & $\mathrm{N}$ & VLI & $\mathrm{N}$ & $\begin{array}{lll}V L & N \\
\end{array}$ & & $V \mathrm{~L}$ \\
\hline C11 & & $\mathrm{VH}$ & $\mathrm{VW}$ & $\mathrm{VL}$ & & $\mathrm{VL} N$ & $\mathrm{VL} N$ & $\mathrm{VL}$ & & $\mathrm{VL}$ & & VL $\mathrm{N}$ & & $\mathrm{VL} / \mathrm{I}$ & $\mathrm{N}$ & $\mathrm{VL}$ & NVL VW & $\mathrm{VH}$ & & $\mathrm{VL}$ & $\mathrm{N}$ & VL I & $\mathrm{N}$ & $\mathrm{VL} N$ & $\mathrm{VI}$ & $V \mathrm{~L}$ \\
\hline $\mathrm{C} 12$ & & $\mathrm{H}$ & $\mathrm{N}$ & $\mathrm{M}$ & & $\mathrm{VL} N$ & $\mathrm{VL} N$ & $\mathrm{VL}$ & & $\mathrm{VH}$ & & $\mathrm{VL} N$ & & $\mathrm{VL}$ & $\mathrm{N}$ & $\mathrm{VL}$ & $\mathrm{N} V L \mathrm{~N}$ & VL & $\mathrm{VW}$ & & $\mathrm{N}$ & & $\mathrm{N}$ & $\mathrm{VL} N$ & VI & $\overline{V L}$ \\
\hline C13 & & $\mathrm{H}$ & $-\mathrm{V}$ & $\mathrm{VL}$ & $-\mathrm{VW}$ & $\mathrm{VL} \mathrm{N}$ & $\mathrm{VL} N$ & $\mathrm{VL}$ & & $\mathrm{VH}$ & & $\mathrm{VL} /$ & & $\mathrm{VL}$ & $\mathrm{N}$ & $\mathrm{VL}$ & $\mathrm{N} / \mathrm{VL} / \mathrm{N}$ & VL & & $\mathrm{VL}$ & $\mathrm{N}$ & VL I & $\mathrm{N}$ & VL V & M & M \\
\hline $\mathrm{C} 14$ & $4 \mathrm{VW}$ & $\mathrm{M}$ & $-\mathrm{VS}$ & $\mathrm{H}$ & $\mathrm{N}$ & $\mathrm{VL} N$ & $\mathrm{VL} / \mathrm{N}$ & $\mathrm{VL}$ & & $\mathrm{H}$ & $\mathrm{N}$ & VL| & & $\mathrm{VL}$ & $\mathrm{N}$ & $\mathrm{VL}$ & $N V L N$ & VL & $\mathrm{N}$ & $\mathrm{VL}$ & $\mathrm{N}$ & VL I & \begin{tabular}{l|l}
$\mathrm{N}$ & $\mathrm{V}$
\end{tabular} & $\mathrm{VL} / \mathrm{N}$ & VI & $V \mathrm{~L}$ \\
\hline
\end{tabular}

Evaluations of the decision maker 3 is given in Table 6 . 
Table 6. Decision maker 3 evaluations

\begin{tabular}{|c|c|c|c|c|c|c|c|c|c|c|c|c|c|c|c|}
\hline & $\mathrm{P}$ & & & $\mathrm{C} 2$ & $\mathrm{C} 3$ & C5 & C6 & C7 & C9 & C10 & $\mathrm{C} 1$ & 11 & \begin{tabular}{|l|l|} 
C12 & C13
\end{tabular} & & C14 \\
\hline & $\mathrm{N}$ & $\mathrm{VL}$ & $\mathrm{N}$ & $\mathrm{VLN}$ & $\operatorname{VL} N \mid \mathrm{VL} N$ & VLNVL & $\mathrm{N}$ & VL NVLN & $\mathrm{VLN}$ & VLN & $\mathrm{VLN}$ & $\mathrm{VL}$ & $\mathrm{N} V \mathrm{~L} N$ & $\operatorname{VL~N}$ & \begin{tabular}{l|l}
$N$ & $V L$ \\
\end{tabular} \\
\hline $\mathrm{C} 1$ & M & $\mathrm{L}$ & N & VLN & $\operatorname{VL} N$ VL $N$ & $\mathrm{VL} / \mathrm{VL}$ & $\mathrm{N}$ & $\mathrm{VL} N \mathrm{NL}-\mathrm{VW}$ & \begin{tabular}{l|l}
$\mathrm{L}$ & $\mathrm{N}$ \\
\end{tabular} & $\mathrm{VL}-\mathrm{S}$ & $\mathrm{VH} N$ & $\mathrm{VL}$ & $\mathrm{NVL} / \mathrm{M}$ & \begin{tabular}{l|l}
$\mathrm{H}$ & $\mathrm{N}$ \\
\end{tabular} & $\mathrm{N} V \mathrm{VL}$ \\
\hline $\mathrm{C} 2$ & $\mathrm{~N}$ & $\mathrm{VL}$ & $\mathrm{N}$ & VLN & VL N VL N & VL NVL & $\mathrm{N}$ & VL N VL N & VLN & $\mathrm{VLN}$ & VL $N$ & $\mathrm{VL}$ & $\mathrm{N} V L-\mathrm{M}$ & $\mathrm{MH}$ & $\mathrm{N}$ VL \\
\hline $\mathrm{C} 3$ & $\mathrm{~V}$ & $\mathrm{VL}$ & & VLN & VLNVL N & $\mathrm{VLNVL}$ & $N$ & VL N VL $N$ & $\mathrm{VL} N \mathrm{~N}$ & VLN & $\mathrm{VL} \mathrm{VS}$ & $\mathrm{SVL}$ & $\mathrm{NVL}-\mathrm{M}$ & $\mathrm{M} V H \mathrm{~N}$ & $\mathrm{~N}$ VL \\
\hline $\mathrm{C} 4$ & $-\mathrm{V}$ & $\mathrm{L}$ & $\mathrm{N}$ & VLN & VL N VL N & VLNVL & $-\mathrm{V}$ & VL N VL N & $\mathrm{VL} N$ & VLN & VL N & $\mathrm{VL}$ & $\mathrm{NVL} / \mathrm{N}$ & VL N & $\mathrm{N} V \mathrm{VL}$ \\
\hline 55 & VS & $\mathrm{H}$ & $\mathrm{N}$ & VLN & VLNL VW & VVLNVL & $\mathrm{N}$ & VL N VL N & VLN & VLN & VL N & $\mathrm{VL}$ & $\mathrm{NVLN}$ & $\operatorname{VLN}$ & $\mathrm{N} V \mathrm{VL}$ \\
\hline 66 & $-\mathrm{M}$ & M & $\mathrm{N}$ & VLN & VL N VH N & VLNVL & $\mathrm{N}$ & VL N VL N & $\mathrm{VL} N$ & VLN & VL N & $\mathrm{VL}$ & $\mathrm{N} V \mathrm{VL}$ & $\operatorname{VLN}$ & $\mathrm{N} V \mathrm{VL}$ \\
\hline 67 & $-\mathrm{S}$ & $\mathrm{VH}$ & & $\mathrm{VL}-\mathrm{S}$ & VL N VL VS & VLNVL & $-\mathrm{VS}$ & $\mathrm{S}$ VHNVLS & VLN $N$ & VLVW & $\mathrm{V} \mathrm{VH}-\mathrm{V}$ & $\mathrm{VVL}$ & $\mathrm{L} / \mathrm{VL} / \mathrm{S}$ & VL N & $\mathrm{N}$ VL \\
\hline 68 & $\mathrm{~N}$ & $\mathrm{~L}$ & N & VLN & $\mathrm{VL} N \mathrm{VL} N$ & VLNVL & $-\mathrm{S}$ & VL $N$ VL $N$ & VL/VW & $\mathrm{VL} \mathrm{V}$ & VL $N$ & $\mathrm{VL}$ & $\mathrm{N} V \mathrm{VL}-\mathrm{S}$ & VL N & $\mathrm{N}$ VL \\
\hline C9 & $\mathrm{N}$ & M & $\mathrm{VS}$ & VLN & VL N VL N & VLNVL & $N$ & VL N VL N & $\mathrm{VL} / \mathrm{N}$ & VLV & $\mathrm{VH} N$ & $\mathrm{VL}$ & $\mathrm{NVL} / \mathrm{S}$ & $\mathrm{VHN}$ & $\mathrm{N} V \mathrm{VL}$ \\
\hline $\mathrm{C10}$ & -VS & $\mathrm{M}$ & N & VLN & VL N VL N & VLNVL & $\mathrm{N}$ & VL N VL N & VLN N & VLN & VL N & $\mathrm{VL}$ & $\mathrm{NVL} / \mathrm{N}$ & $\operatorname{VLN}$ & $\mathrm{N}$ VL \\
\hline $\mathrm{C} 11$ & & $\mathrm{VH}$ & & VLN & $\operatorname{VL} N$ VL $N$ & VLNVL & $N$ & VL N VL N & $\mathrm{VL} N \mathrm{~N}$ & VLN & VL N & $\mathrm{VL}$ & $\mathrm{S} \mathrm{S} V \mathrm{~N}$ & & $-\mathrm{SVL}$ \\
\hline 12 & & $\mathrm{VH}$ & $\mathrm{VS}$ & $\mathrm{MN}$ & VL N VL N & VLNVH & $\mathrm{N}$ & VL N VLN & VLN & VLN & VL N & $\mathrm{VL}$ & $\mathrm{NVL} / \mathrm{N}$ & $\operatorname{VLN}$ & $\mathrm{N}$ VL \\
\hline & & $\mathrm{L}$ & $\mathrm{V}$ & VLN & $\mathrm{VL} N \mathrm{VL} N$ & $\mathrm{VLNL}$ & $\mathrm{N}$ & VL N VLN & VLN N & VLN & VL N & $\mathrm{VL}$ & $\mathrm{NVL} / \mathrm{N}$ & $\mathrm{VL} \mathrm{N}$ & $\mathrm{N}$ VL \\
\hline C14 & & L & $\mathrm{M}$ & \begin{tabular}{l|l}
$\mathrm{H}$ & $\mathrm{N}$
\end{tabular} & VL N VL N & $\mathrm{VLNVL}$ & & VL $N$ VL $N$ & $\mathrm{VL} N \mathrm{~N}$ & VLN & VL N & $\mathrm{VL}$ & $\mathrm{NVL} / \mathrm{N}$ & $\operatorname{VL~N}$ & $\mathrm{N} V \mathrm{VL}$ \\
\hline
\end{tabular}

For the initial vector, it is determined as $[1,1,1,1,1,1,1,1,1,1,1,1,1,1,1]$. After 10 iterations, weights are converged. Results of the application are given in Table 7.

Table 7. Results of the application

\begin{tabular}{|c|c|c|c|c|c|c|c|c|c|c|c|c|c|c|c|}
\hline \multicolumn{2}{|c|}{ Weight } & \multirow{2}{*}{\begin{tabular}{|l|}
$\mathrm{C} 1$ \\
0.599
\end{tabular}} & \multirow{2}{*}{\begin{tabular}{|l|}
$\mathrm{C} 2$ \\
0.642
\end{tabular}} & \multirow{2}{*}{\begin{tabular}{|l|} 
C3 \\
0.659
\end{tabular}} & \multirow{2}{*}{\begin{tabular}{|l|}
$\mathrm{C} 4$ \\
0.930 \\
\end{tabular}} & \multirow{2}{*}{\begin{tabular}{|l|} 
C5 \\
0.749 \\
\end{tabular}} & \multirow{2}{*}{\begin{tabular}{|l|} 
C6 \\
0.637 \\
\end{tabular}} & \multirow{2}{*}{\begin{tabular}{|l|}
$\mathrm{C} 7$ \\
0.659
\end{tabular}} & \multirow{2}{*}{\begin{tabular}{|l|} 
C8 \\
0.645 \\
\end{tabular}} & \multirow{2}{*}{\begin{tabular}{|l|} 
C9 \\
0.659
\end{tabular}} & \multirow{2}{*}{\begin{tabular}{|l|} 
C10 \\
0.857 \\
\end{tabular}} & \multirow{2}{*}{\begin{tabular}{|l|} 
C11 \\
0.676 \\
\end{tabular}} & \multirow{2}{*}{\begin{tabular}{|l|} 
C12 \\
0.659 \\
\end{tabular}} & \multirow{2}{*}{\begin{tabular}{|l|} 
C13 \\
0.645 \\
\end{tabular}} & \multirow{2}{*}{\begin{tabular}{|l|} 
C14 \\
0.659
\end{tabular}} \\
\hline 0.3 & DM1 & & & & & & & & & & & & & & \\
\hline & & & & & & & & & & & & & & & 0.780 \\
\hline & DM3 & & & 659 & & & & & & & & 0.758 & & 0.669 & 0.504 \\
\hline \multicolumn{2}{|c|}{ Aggregated } & 0.808 & 0.596 & & & & & & & & & & 0.701 & 0.624 & 0.633 \\
\hline \multicolumn{2}{|c|}{ Rank } & 2 & 13 & 7 & 1 & 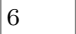 & 14 & 12 & 9 & 8 & 3 & 5 & & & 10 \\
\hline
\end{tabular}

Based on the results, C4-Network infrastructure and C1-Ease of use criteria are determined as first and second most important criteria for the selection of the e-learning systems.

\section{Conclusions}

One of the most effective ways to prevent educational activities from being interrupted in extraordinary situations is to apply for online learning processes. In today's conditions, while all countries in the world struggle with the epidemic disease COVID-19, it has been necessary to apply to e-learning platforms for continuity of educational activities. E-learning becomes an effective tool by combining digital content delivery with learning services. However, at this point, we face the problem of determining which e-learning platform on the market universities should apply. For this reason, it should be investigated which criteria should 
be taken into consideration in choosing the most suitable e-learning platform for educational institutions in selecting the best platform from the alternatives. The most critical point in this process is to apply a priority analysis based on the evaluation criteria for obtaining their rankings. For this aim, we proposed a decision-making approach to obtain the order of importance for the determined criteria to use for the problem of selecting best online education platforms during this pandemic period. We have determined 14 criteria based on the literature research and expert opinions to be used in the process and apply intuitionistic fuzzy cognitive mapping method to determine the most important criterion. As a result of the calculations, it is determined that the most important factor is 'C4-Network infrastructure' and the least important factor in the evaluation of e-learning platforms is 'C6-Exam management system'.

For further studies, the results of the application can be compared with the Pythagorean fuzzy cognitive mapping method's outputs. Also, different scenarios based on the changes in the initial vector can be studied to check its effects.

\section{References}

1. Zare, M., Pahl, C., Rahnama, H., Nilashi, M., Mardani, A., Ibrahim, O., Ahmadi, H.: Multi-criteria decision making approach in E-learning: a systematic review and classification. Appl. Soft Comput. 45, 108-128 (2016)

2. Alptekin, S.E., Karsak, E.E.: An integrated decision framework for evaluating and selecting e-learning products. Appl. Soft Comput. 11(3), 2990-2998 (2011)

3. Begicevic, N., Divjak, B., Hunjak, T.: Prioritization of e-learning forms: a multicriteria methodology. Cent. Eur. J. Oper. Res. 15(4), 405-419 (2007)

4. Bhuasiri, W., Xaymoungkhoun, O., Zo, H., Rho, J.J., Ciganek, A.P.: Critical success factors for e-learning in developing countries: a comparative analysis between ICT experts and faculty. Comput. Educ. 58(2), 843-855 (2012)

5. Chao, R.J., Chen, Y.H.: Evaluation of the criteria and effectiveness of distance e-learning with consistent fuzzy preference relations. Expert. Syst. Appl. 36(7), 10657-10662 (2009)

6. Bo, L., Xuning, P., Bingquan, B.: Modeling of network education effectiveness evaluation in fuzzy analytic hierarchy process. In: 2009 International Conference on Networking and Digital Society, Guiyang, pp. 198-200. IEEE (2009)

7. Axelrod, R.: Schema theory: an information processing model of perception and cognition. Am. Polit. Sci. Rev. 67(4), 1248-1266 (1973)

8. Kosko, B.: Fuzzy cognitive maps. Int. J. Man-Mach. Stud. 24(1), 65-75 (1986)

9. Groumpos, P.P.: Fuzzy cognitive maps: basic theories and their application to complex systems. In: Groumpos, P.P. (ed.) Fuzzy Cognitive Maps, pp. 1-22. Springer, Berlin (2010)

10. Shee, D.Y., Wang, Y.S.: Multi-criteria evaluation of the web-based e-learning system: a methodology based on learner satisfaction and its applications. Comput. Educ. 50(3), 894-905 (2008)

11. Zhang, L., Wen, H., Li, D., Fu, Z., Cui, S.: E-learning adoption intention and its key influence factors based on innovation adoption theory. Math. Comput. Model. 51(11-12), 1428-1432 (2010)

12. Colace, F., De Santo, M., Pietrosanto, A.: Evaluation models for e-learning platform: an AHP approach. In: 36th Annual Conference of Frontiers in Education, San Diego, pp. 1-6. IEEE (2006) 